\title{
CONVEX COMPOSITE FUNCTIONS IN BANACH SPACES AND THE PRIMAL LOWER-NICE PROPERTY
}

\author{
C. COMBARI, A. Elhilali AlaOUi, A. LEVY, R. POLIQUin, AND L. ThiBAult
}

(Communicated by Dale Alspach)

\begin{abstract}
Primal lower-nice functions defined on Hilbert spaces provide examples of functions that are "integrable" (i.e. of functions that are determined up to an additive constant by their subgradients). The class of primal lowernice functions contains all convex and lower- $C^{2}$ functions. In finite dimensions the class of primal lower-nice functions also contains the composition of a convex function with a $C^{2}$ mapping under a constraint qualification. In Banach spaces certain convex composite functions were known to be primal lower-nice (e.g. a convex function had to be continuous relative to its domain). In this paper we weaken the assumptions and provide new examples of convex composite functions defined on a Banach space with the primal lower-nice property. One consequence of our results is the identification of new examples of integrable functions on Hilbert spaces.
\end{abstract}

\section{INTRODUCTION}

In [8], an important class of lower semicontinuous, extended real-valued functions called "primal lower-nice" was introduced. In finite-dimensional spaces, these functions provide interesting and important examples of functions that are "integrable". The integration problem is a very natural one in the theory of non-differentiable functions: a lower semicontinuous function $f$ is deemed integrable if whenever another lower semicontinuous function $g$ satisfies $\partial g(x)=\partial f(x)$ for all $x$, then $f$ and $g$ differ only by an additive constant. Here $\partial$ refers to a subdifferential (i.e. a set of generalized subgradients) which can be taken in many different ways (e.g. Dini subdifferential, Clarke subdifferential, b-subdifferential, Michel-Penot subdifferential, Mordukhovich subdifferential, Ioffe approximate subdifferential, Fréchet subdifferential, and proximal subdifferential). It was well known at the time that convex functions satisfied the integration problem, but very few other examples were known.

The key element in the notion of primal lower-nice functions is that the size of the quadratic needed to "achieve" a proximal subgradient is known and behaves in a linear fashion. Recall that for a lower semicontinuous function $f: \mathbb{R}^{n} \longrightarrow$ $\mathbb{R} \cup\{+\infty\}$, the set of proximal subgradients to $f$ at a point $x \in \mathbb{R}^{n}$, denoted by

Received by the editors February 16, 1996 and, in revised form, November 27, 1996.

1991 Mathematics Subject Classification. Primary 58C20; Secondary $49 J 52$.

Key words and phrases. Primal lower-nice functions, subdifferential, convex composite functions, integrable functions.

The research of R. Poliquin was supported in part by the Natural Sciences and Engineering Research Council of Canada under grant OGP41983. 
$\partial_{p} f(x)$, is the set of points $x^{*} \in \mathbb{R}^{n}$ for which there exist positive scalars $\lambda$ and $T$ such that if $t \geq T$ then the inequality

$$
f\left(x^{\prime}\right) \geq f(x)+\left\langle x^{*}, x^{\prime}-x\right\rangle-(t / 2)\left\|x^{\prime}-x\right\|^{2}
$$

holds for all $x^{\prime}$ within $\lambda$ of $x$. Poliquin [8] calls a function $f$ primal lower-nice at a point $\bar{x} \in \operatorname{dom} f:=\{x \in X: f(x)<+\infty\}$ if there exist $T>0, \lambda>0$, and $c>0$ such that for all points $x$ within $\lambda$ of $\bar{x}$, the proximal subgradient inequality (1) holds for all proximal subgradients $x^{*}$ to $f$ at $x$ satisfying $\left\|x^{*}\right\| \leq c t$ and this for any $t \geq T$. It was shown in [8] that in finite dimensions this property is equivalent to the existence of $\lambda^{\prime}>0, c^{\prime}>0$, and $T^{\prime}>0$ such that

$$
\left\langle x_{1}^{*}-x_{2}^{*}, x_{1}-x_{2}\right\rangle \geq-t\left\|x_{1}-x_{2}\right\|^{2}
$$

whenever $t \geq T^{\prime}, \quad x_{i} \in \bar{x}+\lambda^{\prime} \mathbb{B}_{X}, \quad x_{i}^{*} \in \partial_{p} f\left(x_{i}\right)$, and $\left\|x_{i}^{*}\right\| \leq c^{\prime} t$. In a Hilbert space [7] showed that the two properties outlined above characterize the same class of functions. The relationship between these two properties in a general Banach space has not been addressed until now; we show in Proposition 1.2 that a function which satisfies (1) always satisfies (2). It is not known whether the converse is true in general; however, we are able to establish the converse for a very important class of convex composite functions.

To demonstrate that many important functions have the primal lower-nice property, Poliquin [8] showed that for any lower semicontinuous convex function $f$ : $\mathbb{R}^{m} \longrightarrow \mathbb{R} \cup\{+\infty\}$ and any $C^{2}$-mapping $F: \mathbb{R}^{n} \longrightarrow \mathbb{R}^{m}$, the composition $f \circ F$ is primal lower-nice whenever a natural qualification condition is satisfied. Convex composite functions of the type just described are omnipresent in optimization theory and nonsmooth analysis. In fact the problems most commonly encountered in optimization theory can be reformulated in terms of these functions.

In general Banach spaces Thibault and Zagrodny [10] gave a partial extension of this result; they showed for Banach spaces $X$ and $Y$ that the composition of a lower semicontinuous convex function $f: X \longrightarrow \mathbb{R} \cup\{+\infty\}$ which is continuous relative to its domain with a $C^{2}$-mapping $F: Y \longrightarrow X$ satisfies (2) under the assumption that the Robinson qualification condition holds. (In the finite-dimensional setting, the Robinson qualification condition is equivalent to the qualification condition used by Poliquin [8]). They also showed, in the same paper, that a function defined on a Hilbert space which satisfies (2) is integrable. The main purpose of the present paper is to drop the continuity assumption on the restriction of $f$, weaken the differentiability assumptions on $F$, and show that such functions satisfy not only (2) but also (1) (a result in this direction first appeared in [6]). In doing so we provide new examples of integrable functions on Hilbert spaces and of functions on general Banach spaces that satisfy (1) and (2).

As mentioned at the beginning of this introduction, there are several types of generalized subgradients that can be used. Another added bonus of primal lower-nice functions defined on Hilbert spaces is that all subgradients are the same for these functions (see [7] and [8]). It is not known whether the corresponding statement holds in general Banach spaces; however, we are able to show that in such spaces, all subgradients agree for the convex composite functions used in Theorem 2.4 (i.e. the ones with the weakest assumptions); this result is also part of Theorem 2.4.

Still another interesting property of primal lower-nice functions in finite dimensions is that the subgradient mapping (recall that all subgradients are the same for these functions) is "proto-differentiable", and that the proto-derivative of the 
subgradient mapping is the set of subgradients of the "second-order epi-derivative" (see [9]). Levy, Poliquin, and Thibault [7] gave several partial extensions of this result to primal lower-nice functions on Hilbert spaces. In general Banach spaces, Levy [6, Theorem 3.3] provided an estimate for the "outer graphical derivative" of the subdifferential of primal lower-nice functions (in the sense of (1)) in terms of the subdifferential of the second-order epi-derivative . (Proto-derivatives exist when the inner and outer graphical derivatives coincide.) The results in [6] and [7] can now be applied to the new examples of primal lower-nice functions covered by our Theorem 2.4.

\section{Preliminaries}

In the sequel $X$ and $Y$ will be Banach spaces. For any function $f: X \longrightarrow \mathbb{R} \cup\{+\infty\}$ we will denote by $\partial f$ a generic subdifferential operator for which $\partial f(x)$ is contained in the Clarke subdifferential $\partial_{C} f(x)$ (see [4]). Note that all classical subdifferentials (for example, the Dini subdifferential, Clarke subdifferential, b-subdifferential, Michel-Penot subdifferential, Mordukhovich subdifferential, Ioffe approximate subdifferential, Fréchet subdifferential, and proximal subdifferential) are included in the Clarke subdifferential. The proximal subdifferential plays a special role in this paper, so to indicate the proximal subdifferential operator explicitly, we use the notation $\partial_{p} f$.

Following Poliquin [9], we say that a lower semicontinuous extended real-valued function $f: X \longrightarrow \mathbb{R} \cup\{+\infty\}$ is primal lower-nice at a point $\bar{x} \in \operatorname{domf}:=$ $\{x \in X: f(x)<+\infty\}$ if the proximal subdifferential is nonempty on a dense subset of some neighborhood of $\bar{x}$ intersected with the domain of $f$, and there exist positive scalars $\lambda, c$, and $T$ such that for any $t \geq T$ and any pair $\left(x, x^{*}\right)$ satisfying $\left\|x^{*}\right\| \leq c t,\|x-\bar{x}\| \leq \lambda$, and $x^{*} \in \partial_{p} f(x)$, the inequality (1) holds for all $x^{\prime}$ with $x^{\prime} \in x+\lambda \mathbb{B}_{X}$. (Here $\mathbb{B}_{X}$ denotes the closed unit ball in $X$ centered at the origin.) As in the finite-dimensional case, the inequality (1) is the defining inequality for $x^{*}$ to be a proximal subgradient to $f$ at $x$, so $f$ being primal lower-nice just means that proximal subgradients at points near $\bar{x}$ satisfy the proximal inequality (1) for the same constants $\lambda, c$ and $T$. Note that in any Hilbert space, the proximal subdifferential is automatically nonempty on a dense subset of the domain of $f$, so this component of the definition of a primal lower-nice function did not appear in Poliquin's original version [8].

Obviously, convex functions and lower- $C^{2}$ functions (see [8]) are primal lowernice. Poliquin [8] proved that composite functions defined on finite-dimensional spaces are primal lower-nice whenever a qualification condition is fulfilled. More precisely, Poliquin showed the following result.

1.1. Theorem ([8]). Let $f: \mathbb{R}^{q} \longrightarrow \mathbb{R} \cup\{+\infty\}$ be a lower semicontinuous convex function and $F: \mathbb{R}^{p} \longrightarrow \mathbb{R}^{q}$ be a mapping which is twice continuously differentiable near a point $\bar{x}$ with $F(\bar{x}) \in$ domf. If the qualification condition $N(\operatorname{domf} ; F(\bar{x})) \cap$ $\operatorname{ker}\left(\nabla F(\bar{x})^{*}\right)=\{0\}$ is satisfied (where $\nabla F(\bar{x})^{*}$ denotes the adjoint of $\nabla F(\bar{x})$ and $N(\operatorname{dom} f ;)$ the normal cone to dom $f)$, then $f \circ F$ is primal lower-nice at $\bar{x}$.

The above primal lower-nice property is intimately connected to the proximal subdifferential $\partial_{p}$; however, there is another kind of primal lower-nice property defined in terms of any subdifferential $\partial$. A lower semicontinuous extended realvalued function $f: X \longrightarrow \mathbb{R} \cup\{+\infty\}$ is primal lower-nice at $\bar{x}$ with respect to $\partial$ if the operator $\partial f$ is nonempty on a dense subset of some neighborhood of $\bar{x}$ intersected 
with the domain of $f$, and there exist $\lambda^{\prime}>0, c>0$, and $T>0$ such that (2) holds whenever $t \geq T, \quad x_{i} \in \bar{x}+\lambda^{\prime} \mathbb{B}_{X}, \quad x_{i}^{*} \in \partial f\left(x_{i}\right)$, and $\left\|x_{i}^{*}\right\| \leq c t$. It is always the case that a function $f$ which is primal lower-nice at $\bar{x}$ is primal lower-nice at $\bar{x}$ with respect to the proximal subdifferential. We state this fact as a proposition.

1.2. Proposition. For a Banach space $X$, let $f: X \longrightarrow \mathbb{R} \cup\{+\infty\}$ be a lower semi-continuous function which is primal lower-nice at $\bar{x}$. Then $f$ is primal lowernice at $\bar{x}$ with respect to the proximal subdifferential $\partial_{p}$.

Proof. Let $\lambda, c$, and $T$ be as stipulated by $f$ being primal lower-nice at $\bar{x}$. Choose $x_{1}$ and $x_{2}$ from the ball of radius $\lambda^{\prime}=\lambda / 2$ about $\bar{x}$, and consider any proximal subgradients $x_{1}^{*} \in \partial_{p} f\left(x_{1}\right)$ and $x_{2}^{*} \in \partial_{p} f\left(x_{2}\right)$ satisfying $\left\|x_{i}^{*}\right\| \leq c t$ for $t \geq T$. Since $f$ is primal lower-nice at $\bar{x}$, we have the following inequalities:

$$
\begin{aligned}
& f\left(x_{2}\right) \geq f\left(x_{1}\right)+\left\langle x_{1}^{*}, x_{2}-x_{1}\right\rangle-(t / 2)\left\|x_{2}-x_{1}\right\|^{2}, \\
& f\left(x_{1}\right) \geq f\left(x_{2}\right)+\left\langle x_{2}^{*}, x_{1}-x_{2}\right\rangle-(t / 2)\left\|x_{2}-x_{1}\right\|^{2} .
\end{aligned}
$$

Adding these, we obtain the desired inequality.

When $X$ is a finite-dimensional vector space, Poliquin [9, Proposition 2.2] showed the opposite implication to the one in Proposition 1.2, and Levy, Poliquin, and Thibault [7, Corollary 2.3] extended this equivalence to Hilbert spaces. Note that since limiting proximal subgradients are defined in terms of proximal subgradients, the limiting proximal subdifferential can replace the proximal subdifferential in Proposition 1.2.

Thibault and Zagrodny [10] showed that certain composite functions on infinitedimensional vector spaces are primal lower-nice with respect to the Clarke subdifferential. The composite functions that they studied were infinite-dimensional versions of convex composite functions $f \circ F$ satisfying the constraint qualification

$$
\mathbb{R}_{+}(\operatorname{dom} f-F(\bar{x}))-\nabla F(\bar{x})(X)=Y .
$$

Note that if $\nabla F$ is continuous, $(R)$ holds on a neighborhood of $\bar{x}$; this is an easy application of [5], Theorem 5.2.

1.3. Theorem ([10]). Let $X$ and $Y$ be two Banach spaces. Assume that $F: X \longrightarrow$ $Y$ is twice continuously differentiable at $\bar{x}$, that $f: Y \longrightarrow \mathbb{R} \cup\{+\infty\}$ is convex with $f(F(\bar{x}))<+\infty$, that the restriction of $f$ to dom $f$ is continuous and the qualification condition $(R)$ is satisfied. Then $f \circ F$ is primal lower-nice at $\bar{x}$ with respect to any subdifferential contained in the Clarke subdifferential.

Note that in the finite-dimensional setting the qualification condition in Theorem 1.1 is equivalent to $(R)$, so Theorem 1.3 is a partial extension of Theorem 1.1. In order to establish a complete extension to Banach spaces of Poliquin's theorem [8] (our Theorem 1.1), we will need the following interior mapping theorem by Ursescu [11]. Recall first that for a set-valued mapping $M: U \longrightarrow V$ we define $\operatorname{graph}(M)$ $:=\{(u, v) \in U \times V: v \in M(u)\}, \operatorname{dom} M:=\{u \in U: M(u) \neq \emptyset\}$ and $\operatorname{range}(M)$ $:=M(U)$. Recall also that the core of a convex subset $C$ of $X$ is given by $C o r e C$ $:=\{x \in C: \forall y \in X, \exists \epsilon>0, x+[-\epsilon, \epsilon] y \subset C\}$.

1.4. Theorem (Ursescu). Let $U$ and $V$ be Fréchet spaces and $M: U \longrightarrow V a$ set-valued mapping. Assume that graph $(M)$ is a closed convex subset of $U \times V$ and that core $($ range $M) \neq \emptyset$. Then for any neighborhood $N$ of zero in $U$ one has $M(x) \cap \operatorname{core}($ range $M) \subset \operatorname{int}(M(x+N))$ for every $x \in \operatorname{dom} M$. 


\section{MAIN RESULT}

Let us first establish the following lemmas. For any $r \in \mathbb{R}$ we will use the notation lev $f:=\{x \in X: f(x) \leq r\}$ to denote the $r$-level set of $f$.

2.1. Lemma. Let $X$ and $Y$ be two Banach spaces. Assume that $F: X \longrightarrow Y$ is continuously differentiable at $\bar{x}$, and that $f: Y \longrightarrow \mathbb{R} \cup\{+\infty\}$ is proper convex and lower semicontinuous with $f(F(\bar{x}))<+\infty$. Assume also that the qualification condition $(R)$ is satisfied. Then there exist $r>0, s>0$ such that

$$
s \mathbb{B}_{Y} \subset\left(l e v_{1+f(F(\bar{x}))} f-F(\bar{x})\right) \cap r \mathbb{B}_{Y}-\nabla F(\bar{x})\left(r \mathbb{B}_{X}\right) .
$$

Proof. Let us consider for $\bar{y}:=F(\bar{x})$ the set-valued mapping $M$ from $X \times Y \times \mathbb{R}$ into $Y$ defined by $M(x, y, \lambda)=\{y-\bar{y}-\nabla F(\bar{x})(x)\} \quad$ if $f(y) \leq \lambda$ and $M(x, y, \lambda)=\emptyset$ otherwise. One easily sees that range $M=\operatorname{dom} f-\bar{y}-\nabla F(\bar{x})(X)$.

Then the qualification condition $(R)$ is equivalent to $\mathbb{R}_{+}($range $M)=Y$, and this is equivalent (see [2] ) to $0 \in \operatorname{core}($ range $M)$. Moreover the graph of $M$ is obviously closed and convex. Since $0 \in M(0, \bar{y}, f(\bar{y}))$, we may deduce from the Ursescu theorem that, for every neighborhood $N$ of zero, $0 \in \operatorname{int}(M((0, \bar{y}, f(\bar{y}))+N))$. Let $\left.N:=r \mathbb{B}_{X} \times r \mathbb{B}_{Y} \times\right]-1,1[$. To complete the proof, simply note that there exists $s>0$ such that

$$
\begin{aligned}
s \mathbb{B}_{Y} & \subset M\left(r \mathbb{B}_{X} \times\left(\bar{y}+r \mathbb{B}_{Y}\right) \times\right]-1+f(\bar{y}), 1+f(\bar{y})[) \\
& \subset\left(l e v_{1+f(\bar{y})} f-F(\bar{x})\right) \cap r \mathbb{B}_{Y}-\nabla F(\bar{x})\left(r \mathbb{B}_{X}\right) .
\end{aligned}
$$

2.2. Lemma. Let $X$ and $Y$ be two Banach spaces. Assume that $F: X \longrightarrow Y$ is continuously differentiable at $\bar{x}$, and that $f: Y \longrightarrow \mathbb{R} \cup\{+\infty\}$ is proper, convex and lower semicontinuous with $f(F(\bar{x}))<+\infty$. Assume also that the qualification condition $(R)$ is satisfied. Then for each $k>0$ there exist $c>0, T>0$, and a neighborhood $U$ of $\bar{x}$ such that if $t \geq T, \quad x \in U, y^{*} \in \partial f(F(x))$ and $\left\|y^{*} \circ \nabla F(x)\right\| \leq c t$, then $\left\|y^{*}\right\| \leq k t$.

Proof. Suppose that the statement of the lemma does not hold and set $\bar{y}:=F(\bar{x})$. Then there exist $k>0, c_{n} \searrow 0, t_{n} \nearrow \infty, x_{n} \rightarrow \bar{x}$ and $y_{n}^{*} \in \partial f\left(F\left(x_{n}\right)\right)$ such that $\left\|y_{n}^{*} \circ \nabla F\left(x_{n}\right)\right\| \leq c_{n} t_{n}$ and $\left\|y_{n}^{*}\right\| \geq k t_{n}$. Let $r>0$ and $s>0$ be given by Lemma 2.1, and fix any $b \in \mathbb{B}_{Y}$. By Lemma 2.1 there exist $v \in \operatorname{lev}_{1+f(\bar{y})} f$ with $\|v-\bar{y}\| \leq r$ and $u \in \mathbb{B}_{X}$ such that $s b=v-F(\bar{x})-\nabla F(\bar{x})(r u)$. Then

$$
\begin{aligned}
s\left\langle y_{n}^{*}, b\right\rangle= & \left\langle y_{n}^{*}, v-F(\bar{x})-\nabla F(\bar{x})(r u)\right\rangle \\
= & \left\langle y_{n}^{*}, v-F\left(x_{n}\right)\right\rangle+\left\langle y_{n}^{*}, F\left(x_{n}\right)-F(\bar{x})\right\rangle-r\left\langle y_{n}^{*} \circ \nabla F\left(x_{n}\right), u\right\rangle \\
& \quad-r\left\langle y_{n}^{*} \circ\left(\nabla F(\bar{x})-\nabla F\left(x_{n}\right)\right), u\right\rangle,
\end{aligned}
$$

and hence, since $y_{n}^{*} \in \partial f\left(F\left(x_{n}\right)\right)$, the following inequality holds:

$$
\begin{gathered}
s\left\langle y_{n}^{*}, b\right\rangle \leq f(v)-f\left(F\left(x_{n}\right)\right)+\left\|y_{n}^{*}\right\|\left\|F\left(x_{n}\right)-F(\bar{x})\right\|+r c_{n} t_{n} \\
+r\left\|y_{n}^{*}\right\|\left\|\nabla F(\bar{x})-\nabla F\left(x_{n}\right)\right\| .
\end{gathered}
$$

As $f\left(F\left(x_{n}\right)\right)<+\infty$ (since $y_{n}^{*} \in \partial f\left(F\left(x_{n}\right)\right)$ and $f$ is lower semicontinuous, one has, for $n$ large enough, $F\left(x_{n}\right) \in\left(F(\bar{x})+r \mathbb{B}_{Y}\right) \cap \operatorname{dom} f$ and $f(\bar{y})-1 \leq f\left(F\left(x_{n}\right)\right)$. It follows from (3) and the inequality $f(v) \leq 1+f(\bar{y})$ that the bound

$$
s\left\langle y_{n}^{*}, b\right\rangle \leq 2+\left\|y_{n}^{*}\right\|\left\|F\left(x_{n}\right)-F(\bar{x})\right\|+r c_{n} t_{n}+r\left\|y_{n}^{*}\right\|\left\|\nabla F(\bar{x})-\nabla F\left(x_{n}\right)\right\|
$$


holds, which ensures that the bounds

$$
s\left\|y_{n}^{*}\right\| \leq 2+\left\|y_{n}^{*}\right\|\left\|F\left(x_{n}\right)-F(\bar{x})\right\|+r c_{n} t_{n}+r\left\|y_{n}^{*}\right\|\left\|\nabla F(\bar{x})-\nabla F\left(x_{n}\right)\right\|
$$

and hence

$$
s \leq 2\left(k t_{n}\right)^{-1}+\left\|F\left(x_{n}\right)-F(\bar{x})\right\|+k^{-1} r c_{n}+r\left\|\nabla F(\bar{x})-\nabla F\left(x_{n}\right)\right\|
$$

both hold. Passing to the limit, we get $s=0$, which is a contradiction.

In the next lemma we will compute the Clarke subdifferential of $f \circ F$. A practical way to present this result is to first compute the support function of the Clarke subdifferential of $f \circ F$, which can be defined in terms of the Clarke tangent cone (which is always a closed convex cone). Recall that the Clarke tangent cone $T_{C}(S ; \bar{x})$ to a set $S \subset X$ at a point $\bar{x} \in S$ is the set of all vectors $h \in X$ such that for any sequence $\left\{x_{n}\right\}$ converging to $\bar{x}$ with $x_{n} \in S$ and any scalar sequence $\left\{t_{n}\right\}$ decreasing to 0 , there exists a sequence $\left\{h_{n}\right\}$ converging to $h$ and satisfying $x_{n}+t_{n} h_{n} \in S$ for all $n \in \mathbb{R}$. The support function of the Clarke subdifferential $\partial_{C} g(\bar{x})$ of a function $g: X \rightarrow \mathbb{R} \cup\{+\infty\}$ at $\bar{x} \in$ domg, is the function $g^{\uparrow}(\bar{x} ; \cdot)$ defined by

$$
g^{\uparrow}(\bar{x} ; h)=\inf \left\{r \in \mathbb{R}:(h, r) \in T_{C}(\text { epig; }(\bar{x}, g(\bar{x})))\right\},
$$

where epig $:=\{(x, r) \in X \times \mathbb{R}: r \geq g(x)\}$ is the epigraph of $g$. For any $\bar{x} \in d o m g$, $\partial_{C} g(\bar{x})$ is the subdifferential in the sense of convex analysis of $g^{\uparrow}(\bar{x} ; \cdot)$ at 0 , i.e. $\partial_{C} g(\bar{x})=\left\{x^{*} \in X^{*}:\left\langle x^{*}, h\right\rangle \leq g^{\uparrow}(\bar{x} ; h), \forall h \in X\right\}$. Recall finally that $\partial_{C} g(x)=\emptyset$ for $x \notin$ domg.

2.3. Lemma. Let $X$ and $Y$ be two Banach spaces. Assume that $F: X \rightarrow Y$ is continuously differentiable at $\bar{x}, f: Y \rightarrow \mathbb{R} \cup\{+\infty\}$ is convex lower semicontinuous with $f(F(\bar{x}))<\infty$, and that the constraint qualification $(R)$ is satisfied. Then the Clarke subdifferential of the composition $f \circ F$ is given by

$$
\partial_{C}(f \circ F)(x)=\nabla F(x)^{*} \partial f(F(x))
$$

for all $x$ near $\bar{x}$. Here $\nabla F(x)^{*}$ denotes the adjoint operator of $\nabla F(x)$, and the set on the right side of the above identity is given by

$$
\nabla F(x)^{*} \partial f(F(x)):=\left\{\nabla F(x)^{*} y^{*}: y^{*} \in \partial f(F(x))\right\} .
$$

Proof. Define $F_{0}: X \times \mathbb{R} \rightarrow Y \times \mathbb{R}$ by $F_{0}(x, r)=(F(x), r)$ and note that epi $(f \circ F)=$ $F_{0}^{-1}$ (epif). Observe also that condition $(R)$ ensures that for $\bar{r}=f(F(\bar{x}))$, the identity

$$
\mathbb{R}_{+}(\text {epif }-(F(\bar{x}), \bar{r}))-\nabla F_{0}(X \times \mathbb{R})=Y \times \mathbb{R}
$$

holds. According to Borwein [3, Theorems 4.2 and 5.1], then, for $x \in F^{-1}(\operatorname{dom} f)$ sufficiently close to $\bar{x}$ and $r:=f(F(x))$, the identity

$$
T_{C}(\operatorname{epi}(f \circ F) ;(x, r))=\nabla F_{\circ}(x, r)^{*}\left(T_{C}(\text { epif } ;(F(x), r))\right)
$$

holds. The last identity ensures that for $A=\nabla F(x)$, the following equalities hold:

$$
(f \circ F)^{\uparrow}(x ; h)=f^{\uparrow}(F(x) ; A h)=f^{\uparrow}(F(x) ; \cdot) \circ A(h) .
$$

Moreover, since $\operatorname{dom} f-F(x) \subset \operatorname{dom} f^{\uparrow}(F(x) ; \cdot$ ) (by the convexity of $f$ ), condition $(R)$ also implies that for $x \in F^{-1}(\operatorname{dom} f)$ close enough to $\bar{x}$, the identity $\mathbb{R}_{+}\left(\operatorname{dom}^{\uparrow}(F(x) ; \cdot)-A(X)\right)=Y$ holds. Thus it follows from the equalities (4) and from Aubin and Ekeland [1] that the identity $\partial(f \circ F)^{\uparrow}(0)=A^{*} \partial f^{\uparrow}(F(x) ; \cdot)(0)$ holds. This implies that $\partial_{C}(f \circ F)(x)=A^{*} \partial f(F(x))$. This completes the proof, 
since both sets of the identity in the statement of the lemma are empty for $x \notin$ $F^{-1}(\operatorname{dom} f)$.

2.4. Theorem. Let $X$ and $Y$ be two Banach spaces. Assume that $F: X \longrightarrow Y$ is continuously differentiable at $\bar{x}$ with $\nabla F$ Lipschitz near $\bar{x}, f: Y \longrightarrow \mathbb{R} \cup\{+\infty\}$ is convex lower semicontinuous with $f(F(\bar{x}))<+\infty$, and that the qualification condition $(R)$ is satisfied. Then the composite function $f \circ F$ is primal lower-nice at $\bar{x}$. Moreover, the set of proximal subgradients $\partial_{p}(f \circ F)(\bar{x})$ is identical to the set of Clarke subgradients $\partial_{C}(f \circ F)(\bar{x})$.

Proof. Let $K>0$ be the local Lipschitz constant for $\nabla F$ near $\bar{x}$, fix $k=1 /(2 K)$, and let $c>0, T>0$ and $U$ be as in Lemma 2.2. Choose $\lambda$ small enough so that the set $\bar{x}+(2 \lambda) \mathbb{B}_{X}$ is contained in $U$ as well as in the neighborhood where $K$ is a valid Lipschitz constant for $\nabla F$.

The set of proximal subgradients is always contained in the set of Clarke subgradients, so according to Lemma 2.3, the set $\partial_{p}(f \circ F)(x)$ is contained in the set $\nabla F(x)^{*} \partial f(F(x))$ as long as $x$ is near enough to $\bar{x}$ (we shrink $\lambda$ if necessary to accomplish this). Thus, we will be done if we can show that the primal lower-nice inequality

$$
f\left(F\left(x^{\prime}\right)\right) \geq f(F(x))+\left\langle x^{*}, x^{\prime}-x\right\rangle-(t / 2)\left\|x^{\prime}-x\right\|^{2}
$$

holds whenever $t \geq T, x \in \bar{x}+\lambda \mathbb{B}_{X}, x^{*}=\nabla F(x)^{*} y^{*} \in \nabla F(x)^{*} \partial f(F(x))$ satisfies $\left\|x^{*}\right\| \leq c t$, and $x^{\prime} \in x+\lambda \mathbb{B}_{X}$. Since $y^{*} \in \partial f(F(x))$, the definition of the convex subgradient gives inequality (5) as long as the following inequality holds:

$$
\left\langle x^{*}, x-x^{\prime}\right\rangle+(t / 2)\left\|x-x^{\prime}\right\|^{2} \geq\left\langle y^{*}, F(x)-F\left(x^{\prime}\right)\right\rangle .
$$

To show inequality (6), we consider the point $y^{\prime}$ defined by

$$
y^{\prime}:=\frac{F\left(x^{\prime}\right)-F(x)-\nabla F(x)\left(x^{\prime}-x\right)}{\left\|x-x^{\prime}\right\|},
$$

where we assume that $x^{\prime} \neq x$ (otherwise, inequality (6) holds trivially). By the Mean Value Theorem, the norm of $y^{\prime}$ is bounded above by $K\left\|x^{\prime}-x\right\|$, and according to Lemma 2.2, the norm of $y^{*}$ is bounded above by $k t$. From these two bounds, we obtain the estimate

$$
-(t / 2)\left\|x^{\prime}-x\right\|=-k t K\left\|x^{\prime}-x\right\| \leq-k t\left\|y^{\prime}\right\| \leq\left\langle y^{*}, y^{\prime}\right\rangle,
$$

which by the definition of $y^{\prime}$ gives the desired inequality (6).

As a consequence of the preceding argument, by taking $t \geq T$ large enough, any element of the set $\nabla F(\bar{x})^{*} \partial f(F(\bar{x}))$ is in the set of proximal subgradients $\partial_{p}(f \circ F)(\bar{x})$. According to Lemma 2.3 then, the proximal subgradients $\partial_{p}(f \circ F)(\bar{x})$ must agree with the Clarke subgradients (since proximal subgradients are always Clarke subgradients).

It follows immediately that a function satisfying the conditions of Theorem 2.4 is primal lower-nice with respect to any subdifferential contained in the Clarke subdifferential.

2.5. Corollary. Let $X$ and $Y$ be two Banach spaces. Assume that $F: X \longrightarrow Y$ is continuously differentiable at $\bar{x}$ with $\nabla F$ Lipschitz near $\bar{x}, f: Y \longrightarrow \mathbb{R} \cup\{+\infty\}$ is convex lower semicontinuous with $f(F(\bar{x}))<+\infty$, and that the qualification condition $(R)$ is satisfied. Then the composite function $f \circ F$ is primal lower-nice at $\bar{x}$ with respect to any subdifferential contained in the Clarke subdifferential. 
In the following corollary, we record several applications of our Theorem 2.4 to the integration of primal lower-nice functions and the proto-differentiation of their subgradient mappings. These applications follow directly from [10], [7], and [6] where the definitions of the various generalized derivatives can be found.

2.6. Corollary. Let $X$ and $Y$ be two Banach spaces. Assume that $F: X \longrightarrow Y$ is continuously differentiable at $\bar{x}$ with $\nabla F$ Lipschitz near $\bar{x}, f: Y \longrightarrow \mathbb{R} \cup\{+\infty\}$ is convex lower semicontinuous with $f(F(\bar{x}))<+\infty$, and that the qualification condition $(R)$ is satisfied. If the composition $f \circ F$ is twice strongly epi-differentiable at $\bar{x}$ relative to $\bar{y} \in \partial(f \circ F)(\bar{x})$, then the (strong-weak* ${ }^{*}$ ) outer graphical derivative of the subgradient mapping $\partial(f \circ F)$ is contained in the set of subgradients of the second-order epi-derivative $(f \circ F)_{\bar{x}, \bar{y}}^{\prime \prime} / 2$.

Moreover if $X$ is a Hilbert space, then the function $f \circ F$ is integrable near $\bar{x}$. In this setting, when $f \circ F$ is twice Mosco epi-differentiable at $\bar{x}$ relative to $\bar{y} \in \partial(f \circ F)(\bar{x})$, the subgradient mapping $\partial(f \circ F)$ is proto-differentiable at $\bar{x}$ relative to $\bar{y}$ with proto-derivative equal to the set of subgradients of the function $(f \circ F)_{\bar{x}, \bar{y}}^{\prime \prime} / 2$.

\section{REFERENCES}

[1] J.-P. Aubin and I. Ekeland: Applied nonlinear analysis, Wiley-Interscience (1984). MR 87a:58002

[2] J.M. Borwein, Convex relations in analysis and optimization, in Generalized concavity in Optimization and Economics (S. Schaible and W. T. Ziemba, eds.). Academic Press, New York (1981), 335-376. MR 83g:90075

[3] J.M. Borwein, Stability and regular points of inequality systems, Jour. Opt. Theo. and Appl 48 (1986), 9-52. MR 87m:58018

[4] F.H. Clarke: Optimization and nonsmooth analysis, Wiley, New-York (1983). MR 85m:49002

[5] S. Kurcyusz and J. Zowe, Regularity and stability for the mathematical programming problem in Banach spaces, Appl. Math. Optim. 5 (1979), 49-62. MR 82a:90153

[6] A.B. Levy, Second-order variational analysis with applications to sensitivity in optimization, PhD. Thesis, University of Washington, 1994.

[7] A.B. Levy, R.A Poliquin and L. Thibault, Partial extensions of Attouch's theorem with applications to proto-derivatives of subgradient mappings, Trans. Amer. Math. Soc. 347 (1995), 1269-1294. MR 95k:49035

[8] R.A. Poliquin, Integration of subdifferentials of nonconvex functions, Nonlinear Anal. Th. Meth. Appl. 17(1991), 385-398. MR 92j:49008

[9] R.A Poliquin, An extension of Attouch's theorem and its application to second-order epidifferentiation of convexly composite functions. Trans. Amer. Math. Soc. 332 (1992), 861-874. MR 93a: 49013

[10] L. Thibault and D. Zagrodny, Integration of subdifferentials of lower semicontinuous functions on Banach spaces, J. Math. Anal. Appl. 189 (1995) 33-58. MR 95i:49032

[11] C. Ursescu, Multifunctions with convex closed graph, Czech. Math. J. 7 (25) (1975), 438441. MR 52:8869

(C. Combari, A. Elhilali Alaoui, and L. Thibault) Université de Montpellier II, Laboratoire Analyse Convexe, Place Eugene Bataillon, 34095 Montpellier Cedex 5, France

Current address, A. Elhilali Alaoui: Falculté des Sciences et Techniques de Marrakech, Université Cadi Ayad, B.P. 618, Marrakech, Maroc

(A. Levy) Department of Mathematics, Bowdoin College, Brunswick, Maine 04011

E-mail address: alevy@bowdoin.edu

(R. Poliquin) Department of Mathematical Sciences, University of Alberta, Edmonton, Alberta, Canada T6G 2G1

E-mail address: rene.poliquin@ualberta.ca 